\title{
Timer for use with a Zeiss photocoagulator
}

\author{
P. GAY, K. WIGGINS, AND J. H. DOBREE
}

Departments of Medical Electronics and Ophthalmology, St. Bartholomew's Hospital, London

Photocoagulation, used at the present time for sealing retinal detachments to the under $\vec{\omega}$ lying choroid, and for the destruction of intraocular tumours and of new-formed vessels ino. proliferative diabetic retinopathy, is not without dangers to normal ocular tissues. Thus? it is important to minimize the quantity of light energy used, and moreover it is advantageous to compare results obtained in different types of case, and between different: operators.

The intensity of the light used and the size of the aperture through which it enters the pupil are chosen by the operator, so that the chief variables are the number and duration of the coagulations applied. This timing instrument has been designed to record theses data.

\section{Apparatus}

Referring to the circuit diagram (Fig. I), the timer consists of a squaring amplifier, a gate, and two counters, together with the necessary mains power supply.

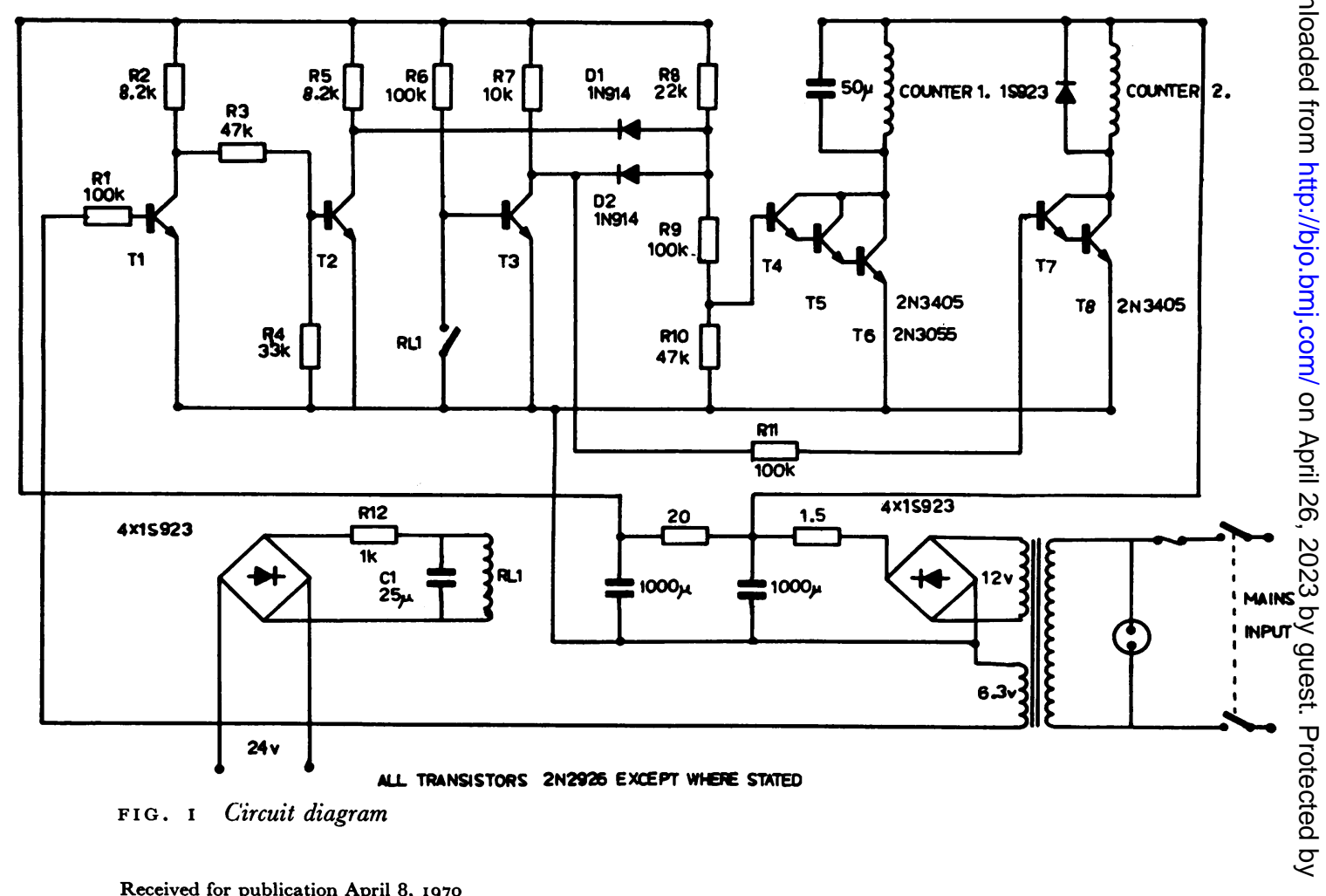


Transistors $T_{1}$ and $T_{2}$ form the squaring amplifier, which uses a mains-derived $6.3 \mathrm{~V} 50 . \mathrm{Hz}$ sine wave to produce square waves which are passed to the AND gate formed by $\mathrm{D}_{1}, \mathrm{D}_{2}$, and R8. The gate is opened by transistor $\mathrm{T}_{3}$ which is turned off by the reed relay $R L_{I}$. RLI is closed by a voltage derived from the photocoagulator shutter-operating voltage, which is rectified by the diode bridge and smoothed by $R_{12}$ and $C_{1}$. The pulses from the gate then pass to $T_{4}, T_{5}$, and $T_{6}$ which step Counter $\mathrm{I}$ at 50 times per second. The closure of $R L_{I}$ also causes $T_{7}$ and $T 8$ to be switched on; thereby giving a count of one on Counter 2.

In operation, each time the coagulation button is pressed, a count of one is recorded on Counter 2 and Counter I counts seconds to the nearest $20 \mathrm{~m} \mathrm{sec}$. Thus the number and total time of the coagulations applied is recorded. After treatment both counters can be manually zeroed ready for the next patient. The instrument is shown in Fig. 2.

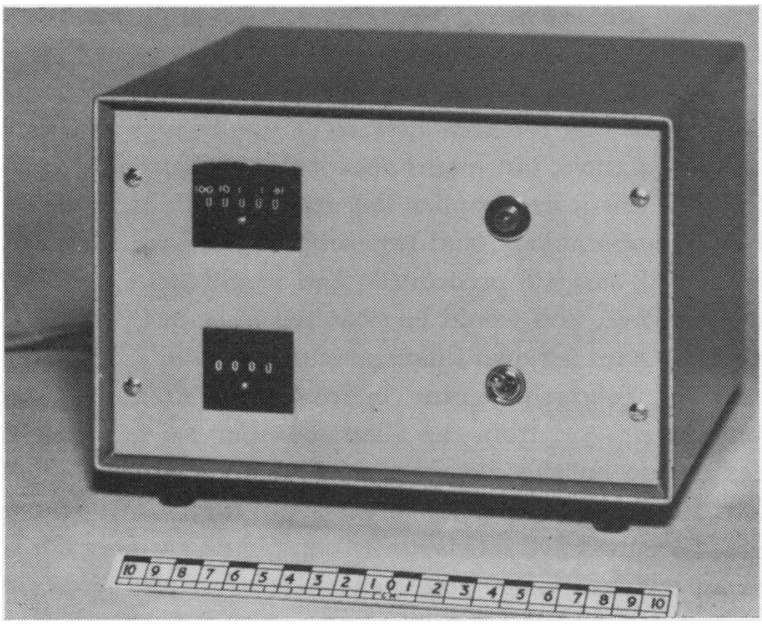

FIG. 2 The Timer. Upper dial records time in seconds. Lower dial records number of coagulations

\section{Summary}

A simple timer has been devised, for use with the Zeiss Photocoagulator, for recording the number and duration of coagulations.

It has now been in use for 2 years and has been found to be of considerable value for recording the dosage given. 